\title{
Spatial relational learning and memory abilities do not differ between men and women in a real-world, open-field environment
}

\author{
Pamela Banta Lavenex* ${ }^{*}$ Pierre Lavenex ${ }^{1}$ \\ Laboratory of Brain and Cognitive Development, Department of Medicine, Unit of Physiology, University of Fribourg, Chemin du Musée 5, 1700 Fribourg, Switzerland
}

\begin{abstract}
This study assesses gender differences in spatial and non-spatial relational learning and memory in adult humans behaving freely in a real-world, open-field environment. In Experiment 1, we tested the use of proximal landmarks as conditional cues allowing subjects to predict the location of rewards hidden in one of two sets of three distinct locations. Subjects were tested in two different conditions: (1) when local visual cues marked the potentially-rewarded locations, and (2) when no local visual cues marked the potentially-rewarded locations. We found that only 17 of 20 adults ( 8 males, 9 females) used the proximal landmarks to predict the locations of the rewards. Although females exhibited higher exploratory behavior at the beginning of testing, males and females discriminated the potentially-rewarded locations similarly when local visual cues were present. Interestingly, when the spatial and local information conflicted in predicting the reward locations, males considered both spatial and local information, whereas females ignored the spatial information. However, in the absence of local visual cues females discriminated the potentially-rewarded locations as well as males. In Experiment 2, subjects ( 9 males, 9 females) were tested with three asymmetrically-arranged rewarded locations, which were marked by local cues on alternate trials. Again, females discriminated the rewarded locations as well as males in the presence or absence of local cues. In sum, although particular aspects of task performance might differ between genders, we found no evidence that women have poorer allocentric spatial relational learning and memory abilities than men in a real-world, open-field environment.
\end{abstract}

\section{Introduction}

A male advantage for spatial abilities as compared to females has been repeatedly reported and is by now relatively well-accepted and considered common knowledge. Historically, gender differences in spatial abilities have been assessed using a variety of paradigms including mental rotation tasks ([1,7,15,40,42,50,53]; see [41] for a review), wayfinding tasks $[16,31,43,44,47]$, environmental pointing tasks [7,23], or object-in-location tasks $[14,19,43,50]$. Interestingly, although each of these paradigms assesses some aspects of spatial cognition, they do not test allocentric spatial memory, the ability to learn and remember a spatial relational representation of the environment independent of the individual's position within that environment.

Research in rodents has typically assessed spatial memory by using tasks such as the Morris water maze (a.k.a., the Morris search task) [35], the Olton 8-arm radial maze [38], or tradi-

\footnotetext{
* Corresponding author. Tel.: +4126300 87 67; fax: +41263009734. E-mail addresses: pamela.bantalavenex@unifr.ch (P. Banta Lavenex), pierre.lavenex@unifr.ch (P. Lavenex).

1 Tel.: +412630087 24; fax: +41263009734
}

tional labyrinth-style mazes [20]. Recently, researchers studying human spatial memory have taken advantage of the development of 3D graphics and computer technology to design tasks which take place in virtual environments, and which aim to emulate the design of these tasks used in animals [2,3,19,33,36,46,51]. Subjects explore these virtual environments by using a joystick to move (i.e., shift their first-person visual perspective) throughout the virtual environment that is displayed on a computer screen. Naturally, researchers have attempted to compare the allocentric spatial relational memory abilities of men and women using these virtual paradigms. Consistent with studies testing mental rotation and wayfinding, these virtual reality studies have identified some gender differences, with men typically performing better than women on a variety of measures such as the latency to find goal locations or complete mazes $[2,3,19,36,46]$, or the number of errors (i.e., wrong turns) made while solving the task [33]. Although it is clear that these studies of spatial abilities in virtual environments demonstrate that men and women perform differentially, what is not clear is (1) whether the measures used specifically assess spatial memory, and thus whether their results can be interpreted to conclude that men have a better memory for spatial locations than women, or (2) whether similar gender differences in allocentric spatial memory exist in real-world (as compared to virtual) environments. 
The establishment of an allocentric representation, or cognitive map, is normally associated with the physical movement of an organism within its environment [37]. Although virtual reality programs have made tremendous progress in simulating the act of moving from a basic visual perspective, there are a number of reasons why virtual environments are fundamentally different from real-world environments for investigating spatial learning and memory abilities. Virtual reality environments are often presented on standard 16-in. ( $41 \mathrm{~cm}$ ) computer screens which, when placed $24 \mathrm{in} .(61 \mathrm{~cm})$ in front of the viewer, allow a field of view of approximately $37^{\circ}$ [51], as compared to nearly $180^{\circ}$ field of view and visual flow in the real world without subjects even moving their head. Furthermore, the virtual environments used in most psychological and brain imaging experiments do not allow subjects access to movement-associated vestibular, proprioceptive, somesthetic, or auditory information. In contrast, these sensory inputs are not only present, but meaningfully coherent with visual input in realworld environments. Whereas all of these sensory inputs are likely integrated when constructing a cognitive map of the environment in the real world $[37,49]$, their absence in a virtual environment makes navigation within that environment fundamentally different, from a functional perspective, to navigation in the real world. Moreover, their absence in virtual environments may differentially affect males and females who may rely on or allot differing weights to various sensory inputs or favor gender-specific cognitive strategies. Additionally, it may also be that the spatial abilities of males and females are differentially influenced by confidence issues with respect to certain types of male-typed tasks (such as spatial tasks; [8-10]), or the manner in which virtual reality tasks are presented (i.e., on a computer). If so, it is possible that the previously-reported gender differences in allocentric spatial relational memory abilities that have derived from experiments in virtual environments are artifacts of the experimental paradigms, or concern specific aspects of visual information processing. As such, it is unclear, and to date has not been tested, whether gender differences in allocentric, spatial relational learning and memory abilities exist in real-world environments.

In order to further investigate possible gender differences in spatial learning and memory abilities, we tested adult male and female human subjects moving freely in a real-world, open-field environment (we use the term "real-world" to contrast specifically with experiments that take place in virtual reality). Our task tests allocentric, spatial relational memory [4,5,24,25], that is, the memory for spatial locations defined in relation to distant environmental cues (i.e., in a frame of reference centered on the objects constituting the environment), and which is therefore independent from the subject's own location or orientation (i.e., not centered on the subject's body position or egocentrically). Importantly, allocentric spatial memory has been shown in rodents and non-human primates to be dependent on the integrity of the hippocampal formation $[4,5,20,34,39]$. Furthermore, this task tests spatial learning and memory in a controlled, real-world environment in which subjects have access to all sensory information normally available when moving about in everyday life $[30,48]$.

\section{Materials and methods}

Human subjects research was approved by the Intercantonal Ethics Committee for Jura, Neuchatel, Fribourg (Neuchatel, Switzerland), and was in accordance with the NIH guidelines for the use of human subjects in research.

\subsection{Experiment 1}

\subsubsection{Experimental subjects}

Subjects were 20 adults (10 males, age range $25-49$ years, average 32.8 years; 10 females, age range $25-51$ years, average 32.5 years). Subjects were students, faculty and staff recruited from the Department of Medicine at the University of
Fribourg, Switzerland. Subjects were tested for approximately 30 min each day for 5 consecutive days (Monday through Friday), between 7 A.M. and 6 P.M.

\subsubsection{Testing room and arena}

Testing took place in a large rectangular room $(6 \mathrm{~m} \times 14 \mathrm{~m}$; Fig. 1) containing many polarizing features such as doors, windows, tables, chairs, and wall posters. The $4 \mathrm{~m} \times 4 \mathrm{~m}$ testing arena was located in one end of the room. Poster display boards (their bottom half covered with paper) were used to construct three boundary walls designating the arena. On the two lateral sides of the arena the poster display boards were placed approximately one meter from the room walls; on the third side, the boards were placed directly adjacent to the room wall. The fourth boundary, between the testing arena and the experimenter, was delineated by a rope attached to the two opposing poster boards, and suspended $50 \mathrm{~cm}$ off the ground. At each of the four near and far corners of the side walls was a $60-\mathrm{cm}$ gap that served as one of the four different entry points through which the subjects must pass in order to enter and exit the testing arena. When subjects were not in the testing arena (i.e., during the intertrial interval), they sat in a chair behind one of the two arena side walls with their back facing the arena and thus could not see where rewards were being placed. From within the arena, and from the intertrial waiting area, subjects had access to distant visual cues within the room.

The testing arena was void of all objects except for 18 plastic cups which were placed inverted on 18 paper plates that were regularly arranged in two hexagonal patterns (an inner hexagon and an outer hexagon) on the floor (Fig. 1). The linoleum floor of the testing arena was entirely uniform, and thus could not provide the subjects with any local landmarks during the task. Because the plastic cups were inverted, the subjects had to lift them or turn them over to obtain a food reward hidden beneath. Two different conspicuous objects could be placed at the center of the arena (the central objects; Fig. 1B and C); the presence of each object was predictive of the presence of food in one of two sets of three distinct locations (Fig. 2, see below). All testing was videotaped with a video camera located in front of the testing arena.

\subsubsection{Procedure}

This exact same task has been previously used to test the spatial relational learning and memory abilities of macaque monkeys [4,24,25]. Thus, in order to make the comparisons between the human and monkey results as valid as possible, human subjects were given no specific instructions as to the goal of the task or what they should do in the arena. Subjects were only told that they would be participating in a memory test that would involve searching for food rewards. On the first day, subjects were seated at a table at the opposite end of the room from the arena where they watched as a food reward (subjects could choose to search for either salted peanuts or Smarties ${ }^{\mathrm{TM}}$ ) was placed underneath an inverted cup. Subjects were then told that they could retrieve the reward, which they all did. Following this single pretraining trial, subjects were led to one of the two intertrial waiting areas on the sides of the arena and told to sit in the chair with their back to the arena until they were called with a number (1 through 4 ) which indicated the door through which they were to enter the arena (denoted by a piece of paper hanging next to the opening with the specific number on it). They were also told that after some unspecified time in the arena another number would be called (1 through 4 ), signaling that they should exit via the specified door number (numbers were also placed next to the four openings on the inside of the arena) and sit in the chair on that side of the arena until called upon to re-enter the arena once again. Subjects were allowed as much time in the arena as they wished, but were called out (i.e., an exit door verbally signaled) $10 \mathrm{~s}$ after they had decided to stop searching. Subjects were requested not to speak to other subjects regarding specifics of the task until testing of all subjects was completed. Two different testing schedules were employed. The 10 subjects ( 5 males and 5 females) tested with schedule 1 received 15 trials per day for 5 consecutive days. The 10 subjects ( 5 males and 5 females) tested with schedule 2 received 10 trials per day for 5 consecutive days. Since no statistical differences were found between the results of subjects tested with these differing schedules (not shown), their results were combined and analyzed together (see below for more detail).

The task required subjects to find the rewards that were located in one of two different arrays of three distinct locations (Fig. 2; either the outer array, locations 4 $8,12$, or the inner array, locations $13,15,17)$. When the outer array was rewarded, all three locations (i.e., 4, 8 and 12) were rewarded simultaneously. Similarly, when the inner array was rewarded, all three locations (i.e., 13, 15 and 17) were rewarded simultaneously. From the intertrial waiting area, subjects were unable to see the arena or the cups while they were being replenished by the experimenter. Furthermore, while the rewards were being replenished between trials, the experimenter lifted and replaced at least six additional cups within the arena (i.e., in addition to the three actually being rewarded) so that subjects could not use auditory cues to determine which, nor how many, locations would be rewarded.

The rewarded array of three distinct locations changed pseudo-randomly between trials. For each trial, a distinct object that could be associated with the presence of rewards in each of the two different arrays was placed at the center of the arena, and could thus be used to predict which array of three distinct locations was rewarded (see below). An inverted yellow cylindrical bin $(19 \mathrm{~cm}$ in diameter $\times 21.5 \mathrm{~cm}$ high) was associated with the presence of rewards at locations 4,8 and 12 on the outer array (Fig. 2). An inverted green rectangular bucket ( $21 \mathrm{~cm} \times 21 \mathrm{~cm} \times 27 \mathrm{~cm}$ high) was associated with the presence of rewards at locations 13,15 and 17 on the inner array. On Day 4 of the experiment, the relationship 
(A)

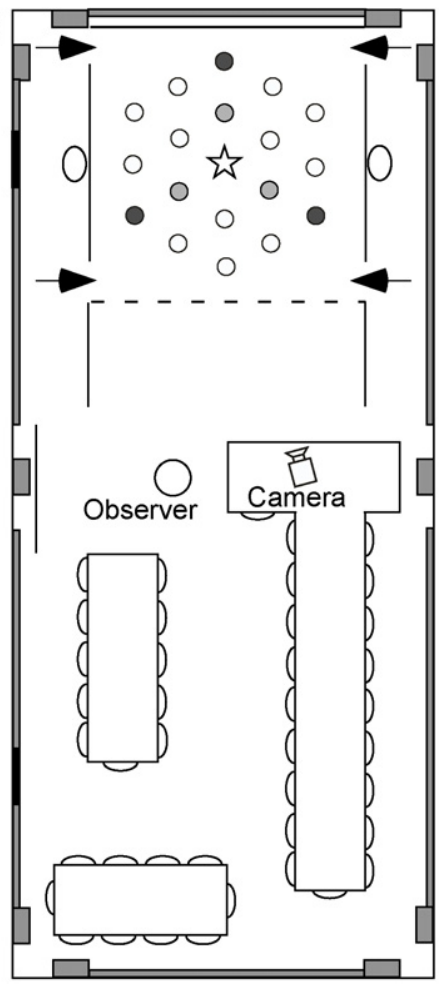

(B)

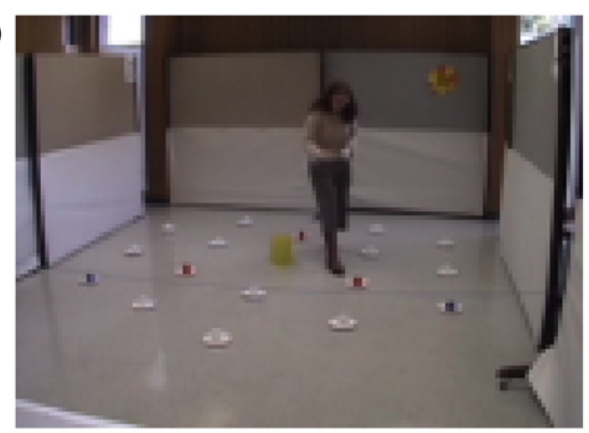

(C)

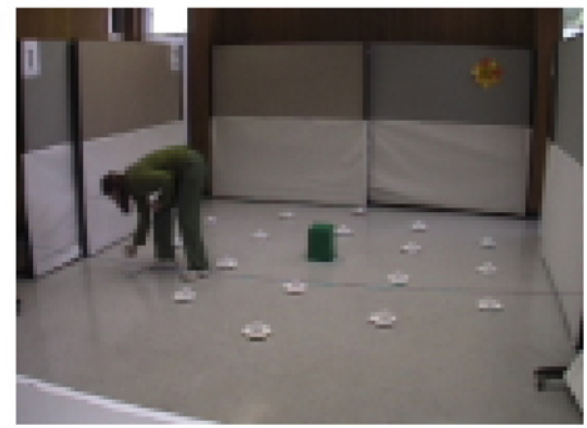

Fig. 1. The testing environment for Experiment 1. (A) Schematic aerial view of the experimental room $(6 \mathrm{~m} \times 14 \mathrm{~m})$ containing polarizing features such as doors (black rectangles), windows, tables (white rectangles), chairs, and wall posters (not shown). Poster display boards (solid lines) defined three of the boundary walls designating the arena. At each of the four near and far corners of the side walls was a $60-\mathrm{cm}$ gap that served as one of the four different entry points (arrows) through which the subjects must pass in order to enter and exit the testing arena. (B) Picture of a subject in the arena during a local cue trial (specific trial is from Day 4, following the reversal manipulation). Note the red and blue colored cups at locations 4, 8, 12,13, 15 and 17. (C) Picture of a subject in the arena during a spatial relational trial (specific trial is from Day 5 , following the reversal manipulation). Note that all locations are covered by identical white (neutral) cups. See main text for detailed description of the experimental room and procedure. (For interpretation of the references to color in this figure legend, the reader is referred to the web version of the article.)

between the central object and the rewarded array was reversed. Thus, for Day 4 and Day 5 , the presence of the cylindrical yellow central object signaled the presence of rewards on the inner array (locations 13,15, and 17), whereas the presence of the rectangular green central object signaled the presence of rewards on the outer array (locations 4,8 , and 12). The reversal of the central object/rewarded array relationship allowed us to further identify which subjects were using the central object as a non-spatial relational cue, because subjects using the cue should choose to search locations on the incorrect array on the first trial in the reversed condition. We further assessed whether subjects relied on the central object to predict rewarded locations by analyzing subjects' first choice upon entering the arena on the specific trials of Days 4 and 5 during which the central object and the rewarded array were different from those used in the immediately preceding trial. We performed Chi-square analyses on the number of correct versus incorrect choices. The number of trials that were analyzed thus depended on the testing schedule. For subjects tested with schedule 1 , four trials were available for analysis. For subjects tested with schedule 2 , eight trials were available for analysis.

2.1.3.1. Testing. The experiment consisted of two separate conditions, the local cue condition and the spatial relational condition, and included two additional probe trials.

2.1.3.2. Local cue condition. On the 1 st, 2 nd and 4 th days of testing, subjects were tested to determine their ability to find rewards at spatially fixed locations marked by local cues, i.e., by red and blue colored cups. In this condition, subjects could find and remember the rewarded locations either by associating the presence of the local cues with the rewarded cups, or by remembering the spatial locations of the rewarded cups based on their relation to distant environmental cues. The subjects were also free to learn and remember the relationship between the central object and the specific rewarded array. Specifically, when the yellow central object was present, the three blue cups located at positions 4,8 and 12 on the outer array were rewarded. When the green central object was present, the three red cups located at positions 13,15 and 17 on the inner array were rewarded (NB: These relations were reversed on the 4 th and 5 th days of testing.). The particular array that was rewarded changed pseudo-randomly between trials.

2.1.3.3. Dissociation probe trials. The first trial of Days 3 and 5 were probe trials during which the locations of the colored cups were shifted $60^{\circ}$ from their usual spatial locations and none of the locations were rewarded (Fig. 2B). Probe trials were followed immediately (after a 1-min intertrial interval) by spatial relational trials in which all 18 cups were the same neutral color, white (see below). The aim of these probe trials was to assess the subjects' reliance on local versus spatial relational information to discriminate the potentially-rewarded locations from the never-rewarded locations. During local cue trials, potentially-rewarded locations were covered by a colored cup so that both local and spatial information were coherent (Fig. 2A). During the dissociation probe trial (Fig. 2B), the colored cups covered the never-rewarded locations $2,6,10,14,16$, and 18 and neutral cups covered the normally potentially-rewarded locations $4,8,12,13,15$, and 17 . As subjects entered the arena, they encountered the same pattern of cups distributed throughout the arena, but the absolute locations of the colored cups within the arena were changed, thus rendering the local and spatial relational information incoherent. The subjects were thus faced with two competing strategies to determine where the food rewards might be hidden, either (1) under the colored cups at spatially "incorrect" locations, or (2) under white (neutral) cups at "correct" spatial locations.

2.1.3.4. Spatial relational condition. Immediately following the dissociation probe trial on Days 3 and 5 , subjects were tested in the spatial relational condition in which no local cues (i.e., no red or blue colored cups) marked the potentially-rewarded locations (all 18 cups were white in color; Figs. 1C and 2C). In this case, the subjects could not discriminate between potentially-rewarded and never-rewarded locations based on local features. Instead, subjects had to rely on an allocentric, spatial relational representation of the environment to discriminate these locations (i.e., coding the goal locations in relation to distant environmental cues). The objects placed at the center of the arena could still be used to predict which of the two arrays of three distinct locations was rewarded. Results from both days were included in the analyses.

\subsubsection{Data analysis}

Because a subject's latency to solve a task might be influenced by many different factors such as confidence, strategy, and motivation (i.e., the learningperformance distinction problem $[13,45,52]$ ), we do not rely on latency as a measure of spatial memory ability. Instead, we determine whether subjects are accurate at finding the rewards by determining whether and how well they discriminate potentially-rewarded locations from never-rewarded locations, thus demonstrating that subjects "know" where the rewards are hidden [30]. 
To assess subjects' ability to discriminate the potentially-rewarded locations from the never-rewarded locations in the local cue and spatial relational conditions, each location was classified into one of five categories with respect to whether it was potentially rewarded and its position in the open-field arena: "Pot IN" designated the three potentially-rewarded locations at the corners of the inner hexagon (Fig. 2; locations 13, 15, 17); "Pot OUT", the three potentially-rewarded locations at the corners of the outer hexagon (locations 4, 8, 12); "Equ IN", the three neverrewarded locations at the corners of the inner hexagon (locations 14,16, 18; denoted "equivalent" because of their position at one of the three corners of the hexagon topologically equivalent to the position of the potentially-rewarded locations); "Equ OUT", the three never-rewarded locations at the corners of the outer hexagon (locations 2, 6, 10); and "Other", the never-rewarded locations on the sides of the outer hexagon (locations $1,3,5,7,9,11$ ). Analyzing subject choices with respect to these five categories allows us to characterize the strategies that subjects rely upon to

(A)

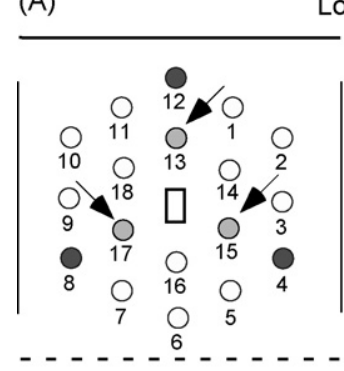

Local Cue

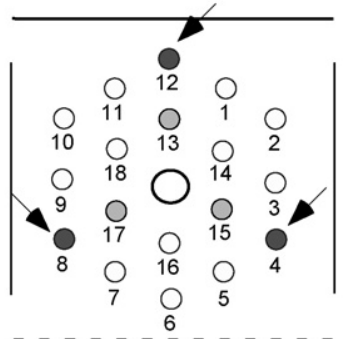

(B)

Probe Trial
(C)

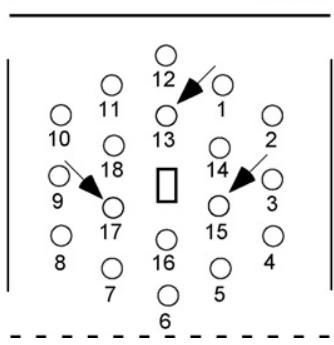

discriminate the rewarded locations $[4,24,25]$. In the spatial relational condition, subjects must remember these locations in relation to distant environmental cues (i.e., they must form a spatial relational representation of the environment) in order to discriminate Pot IN from Equ IN locations, or Pot OUT from Equ OUT locations, which are located at topologically equivalent locations at the corners of the inner and outer hexagon, respectively. For each analysis, we normalized the number of choices of a particular type based on the probability to make that choice. The choices of Pot IN (locations 13, 15, 17), Pot OUT (locations 4, 8, 12), Equ IN (locations 14, 16 , 18) and Equ OUT (locations 2, 6, 10) were divided by three and the number of Other choices (locations $1,3,5,7,9,11$ ) was divided by six.

Only the first 10 trials were used for statistical analyses (thus omitting the last 5 daily trials for subjects tested with schedule 1 that had 15 trials per day). For the local cue and spatial relational trials, we report the first and the first three choices made (i.e., cups opened) by subjects upon entering the arena. For the dissociation probe trial we report the first three choices made by subjects. Because subjects developed unique strategies to find the rewarded locations, we also analyzed the choices made by each individual to detect evidence of spatial relational learning and memory. In this case, statistical analyses were performed within subject across daily sessions. SPSS 16.0 statistical software was used to perform statistical analyses.

\subsubsection{Task specificity}

Because the importance of distinguishing between egocentric and allocentric spatial strategies has been overlooked in some of the literature investigating spatial memory in primates (see $[4,5,24,25]$ for a review) it is worth explaining how the use of four different entrance points and multiple reward locations distributed among 18 total locations prohibits subjects from using egocentric strategies, i.e., strategies based on identifying the goal location in relation only to the subjects body, in order to solve the open-field task [4,5,24,25]. For example, a subject using an egocentric strategy to identify rewarded locations, such as "first cup on my right," would correctly identify only one of the six rewarded locations (i.e., location 4 when entering from the nearest entrance point). However, when entering from any other entrance, the subject would be incorrect. One could argue, then, that subjects could encode 4 different egocentric rules for how to find one rewarded location from each entrance point (after which, the other rewarded locations could be identified by counting the number of cups between rewarded locations). However, in order for this strategy to work, the subject must be able to apply each of the four egocentric rules from the appropriate entry point (because each entry has its own separate egocentric rule, how would one determine which rule is appropriate for which entry in order to choose correctly?). Indeed, the only way to apply the correct egocentric cue would be to have encoded the entrance location in a framework that is independent of the position of the subject's body, i.e., in an allocentric, spatial relational representation (i.e., the entrance at the front of the arena near the wall with the windows, not near the wall with the doors ... a position that is invariant regardless of where the subject is within the room). Thus, whether subjects rely entirely on an allocentric representation to identify reward locations, or whether they employ a strategy that combines both allocentric and egocentric components, it is clear that to identify the rewarded locations in this open-field arena subjects must learn and remember an allocentric, spatial relational representation of the environment.

\subsection{Experiment 2}

The general procedures and testing conditions were similar to those used in Experiment 1, but for this experiment we were interested in testing male and female subjects with only 3 pseudo-randomly arranged reward locations among the 18 possible locations. As the experiment was focused exclusively on testing allocentric spatial relational abilities, we no longer included a central object in the arena, and thus all three rewarded locations were consistently rewarded on every trial (in contrast to Experiment 1 where rewarded locations alternated between the inner and outer arrays). Below we describe the methodological details that differed from Experiment 1.

\subsubsection{Experimental subjects}

Subjects were 18 adults ( 9 males, age range 21-24 years, average 22 years; 9 females, age range 21-26 years, average 23 years). Subjects were bachelor students in Biomedical Sciences at the University of Fribourg, Switzerland. Subjects were tested in one daily session of approximately 45 min between 7 A.M. and 6 P.M.

\subsubsection{Testing room and arena}

Testing took place in a large room $(6 \mathrm{~m} \times 6 \mathrm{~m})$ containing many polarizing features such as doors, windows, tables, chairs, and wall posters. The overall organization was similar to that described for Experiment 1.

The testing arena was the same as for Experiment 1, except for the fact that 18 identical grey plastic disks (foot pads), each with 6 L.E.D. lights arranged in a regular fashion on the top outer edge of the disks, were regularly arranged on the floor as described in Experiment 1. Three of the foot pads could illuminate when touched lightly with the foot. The floor of the room was covered with a uniform blue carpet, and thus could not provide the subjects with any local landmarks during the task In contrast to Experiment 1 , subjects did not have to bend down to find a reward; 
instead they simply needed to touch the foot pads to turn the light on. The lights stayed on for one minute once they had been activated.

\subsubsection{Testing procedure}

Subjects were told that they would be participating in a search task, and that their goal was to find all of the disks that illuminated. Subjects were shown a foot pad and asked to illuminate it themselves with their foot before the experiment started. Locations 7, 10 and 14 served as the goal locations (i.e., the disks at these locations would light up) for all trials for all participants. In contrast to Experiment 1 , no object was present at the center of the apparatus. Subjects were tested for 26 trials in two alternating conditions: 13 trials in the local cue condition (trials 1 , $3,5,7, \ldots, 25$ ), and 13 trials in the spatial relational condition (trials $2,4,6,8, \ldots$, $26)$. In the local cue condition, white plastic disks surrounded the three illuminating goal locations $(7,10,14)$. All other locations were unmarked. In the spatial relation condition, no local cues marked the illuminating goal locations. One final trial, trial 27 , was the dissociation probe trial, during which the white local cues were placed at locations 3,12 and 17, but no disks would illuminate when stepped on

\subsubsection{Data analysis}

To assess subjects' ability to discriminate the goal locations from the Other locations, we distinguished between "correct" (illuminating foot pads) and "incorrect" (non-illuminating foot pads) locations. We normalized the number of choices of a particular types based on the probability to make that choice: The number of times that a correct location was chosen was divided by 3 , and the number of times that an incorrect location was chosen was divided by 15 . For the probe trials, we distinguished "Space" locations $(7,10,14)$, "Color" locations $(3,12,17)$, and "Other" locations. The number of Space and Color location choices was divided by 3 , and the number of Other location choices was divided by 12 . Other analyses and statistical procedures were the same as in Experiment 1.

\section{Results}

\subsection{Experiment 1}

\subsubsection{Number of cups opened}

We first analyzed the total number of cups opened by male and female subjects in order to detect potential gender differences in overall task performance. Interestingly, we found that on Day 1, although the total number of cups opened by males and females decreased similarly across trials (trials 1-10: $F_{(9,162)}=14.818$, $P<0.0001$; interaction: $\left.F_{(9,162)}=1.103, P=0.364\right)$, female subjects opened, on average, almost twice as many cups as male subjects (Fig. 3A; gender: $F_{(1,18)}=10.548, P=0.004$; males: $6.90 \pm 1.26$ (mean \pm SE); females: $12.69 \pm 1.26$ ). This difference was no longer present on Days 2, 3, 4 or 5 (Day 2: $F_{(1,18)}=2.470, P=0.133$; Day 3: $F_{(1,18)}=1.165, P=0.295$; Day 4: $F_{(1,18)}=1.185, P=0.291$; Day 5: $F_{(1,18)}=0.705, P=0.412$; data not shown).

Further detailed analyses revealed, however, that although females opened more cups overall than males on Day 1 , this did not reflect an inability to identify the potentially-rewarded locations. Indeed, males and females did not differ in the number of cups that they opened in order to find all three rewards (Fig. 3B; $F_{(1,18)}=2.977$, $P=0.102$; males: $4.38 \pm 0.42$; females: $5.40 \pm 0.42$ ). In sum, females opened more cups than males only after the third reward was found $\left(F_{(1,18)}=6.258, P=0.022\right.$; males: $2.52 \pm 1.34$; females: 7.271 .34 ; data not shown).

\subsubsection{Local cue condition}

We then evaluated the subjects' ability to discriminate the potentially-rewarded locations in the presence of local cues marking these locations (Days 2 and 4). As described in Section 2, locations were classified into one of the five following categories: "Pot IN" (Fig. 2; locations 13, 15, 17); "Pot OUT" (locations 4, 8, 12); "Equ IN" (locations 14, 16, 18); "Equ OUT" (locations 2, 6, 10); and "Other" (locations 1, 3, 5, 7, 9, 11). Analyzing subject choices with respect to these five categories allows us to characterize the strategies that subjects rely upon to discriminate the rewarded locations $[4,24,25]$.

For their first choice (i.e., the first cup opened during an individual trial), both adult male and female subjects
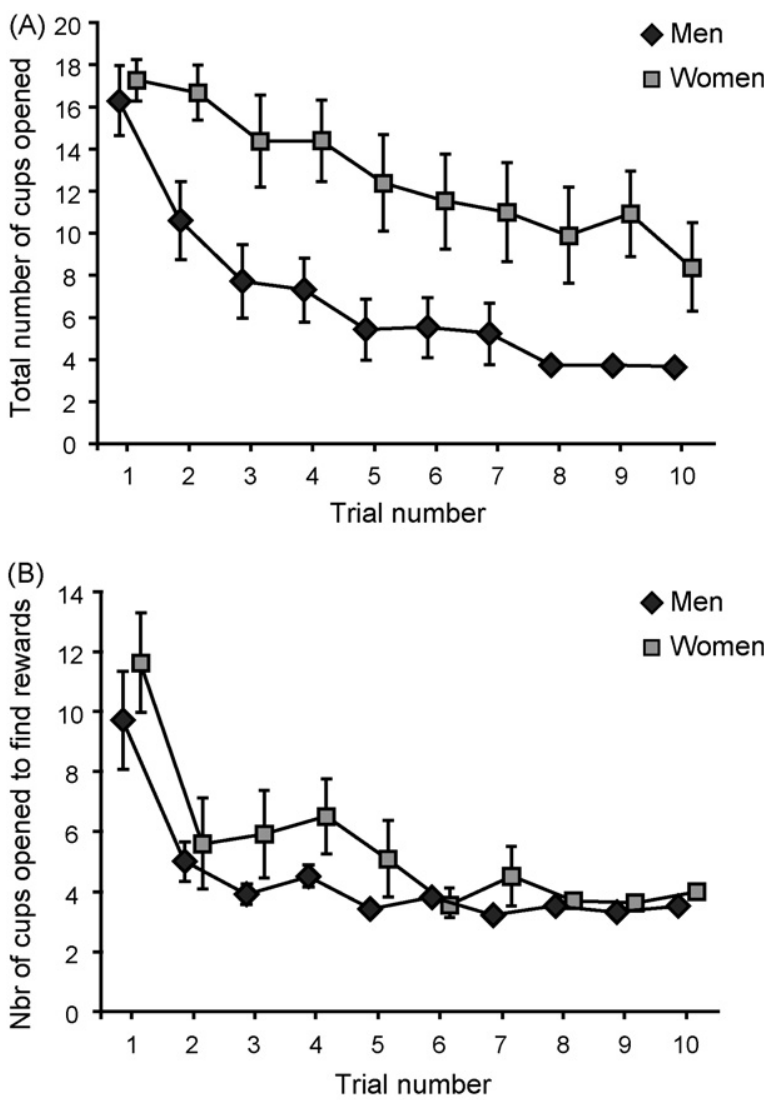

Fig. 3. Number of cups opened by males and females in the first 10 trials of Day 1 in the local cue condition of Experiment 1. (A) Total number of cups opened per trial. (B) Number of cups opened to find the three rewards per trial.

chose potentially-rewarded locations more often than neverrewarded locations (Fig. 4A; gender: $F_{(1,18)}=0.094, P=0.763$; cup choice: $F_{(4,72)}=509.634, P<0.0001$; Pot IN $=$ Pot OUT $>$ Equ IN $=$ Equ OUT $=$ Other, all $P<0.0001$; interaction: $\left.F_{(4,72)}=0.179, P=0.949\right)$. For their first three choices (i.e., the first three cups opened during an individual trial), males and females continued to choose potentially-rewarded locations more often than never-rewarded locations (Fig. 4B; gender: $F_{(1,18)}=1.851, P=0.190$; cup choice: $F_{(4,72)}=1102.853, P<0.0001$; Pot IN = Pot OUT $>$ Equ IN = Equ OUT, all $P<0.0001$; Pot IN $=$ Pot OUT $>$ Other, all $P<0.0001$; Other $>$ Equ In, $P=0.041$; interaction: $\left.F_{(4,72)}=1.309, P=0.275\right)$. Analyses of each individual subject's choices in the local cue condition confirmed that all 20 subjects were equally motivated and discriminated the potentially-rewarded from the never-rewarded locations (data not shown). In sum, in the local cue condition, all subjects readily discriminated the two sets of potentially-rewarded locations marked by different local cues (red and blue cups). Both male and female subjects were highly selective and opened almost exclusively potentially-rewarded cups at fixed locations marked by local cues.

\subsubsection{Probe trials}

In order to assess subjects' reliance on local versus spatial relational information to discriminate potentially-rewarded locations from never-rewarded locations, the first trial of Days 3 and 5 was a probe trial during which the locations of the colored cups were shifted $60^{\circ}$ from their usual spatial locations and none of the locations were rewarded (Fig. 2B; see Section 2). Probe trials were followed by standard spatial relational trials (see below). For the probe trial analyses, locations were classified into one of three categories: "Space" designated the six previously 
potentially-rewarded locations (although there were no rewards present during the probe trials), "Color" designated the six locations now covered by colored cups, and "Other" designated the six locations that were never rewarded and not covered by colored cups.

Examination of the first three cups opened during the probe trial on Day 3 (before subjects had experienced the arena in the absence of local cues) revealed that both males and females preferred colored cups at incorrect spatial locations (Color) as compared to neutral cups at previously-rewarded correct spatial locations (Space) and neutral cups at never-rewarded locations (Other; Fig. 5A; gender: $F_{(1,18)}=0.0, P>0.999$; cup choice: $F_{(2,36)}=30.228, P<0.001$; Color $>$ Space $=$ Other, all $P<0.0001$; interaction: $\left.F_{(2,36)}=0.685, P=0.511\right)$. During the probe trial on Day 5 (following 1 day of experience in the absence of local cues, Day 3 , and another day in the presence of local cues, Day 4), both males and females still demonstrated a preference for colored cups at incorrect spatial locations for the first cup opened (data not shown). However, cup choice for the first three cups opened differed between males and females (gender: $F_{(1,18)}=0.0, P>0.999$; cup choice: $F_{(2,36)}=25.364, P<0.001$; interaction: $F_{(2,36)}=5.727$, $P=0.007)$. Interestingly, males demonstrated equal preference for incorrect, never-rewarded spatial locations covered by colored cups (Color) and previously-rewarded correct spatial locations (Space), and preferred both types of locations to never-rewarded locations covered by neutral cups (Other; Fig. 5B; $F_{(2,18)}=8.647$, $P=0.002$, Color $=$ Space $>$ Other, all $P<0.045)$. Female subjects, in contrast, still demonstrated a preference for incorrect spatial locations covered by colored cups (Color), and even chose other, never-rewarded locations (Other) more often than previously-
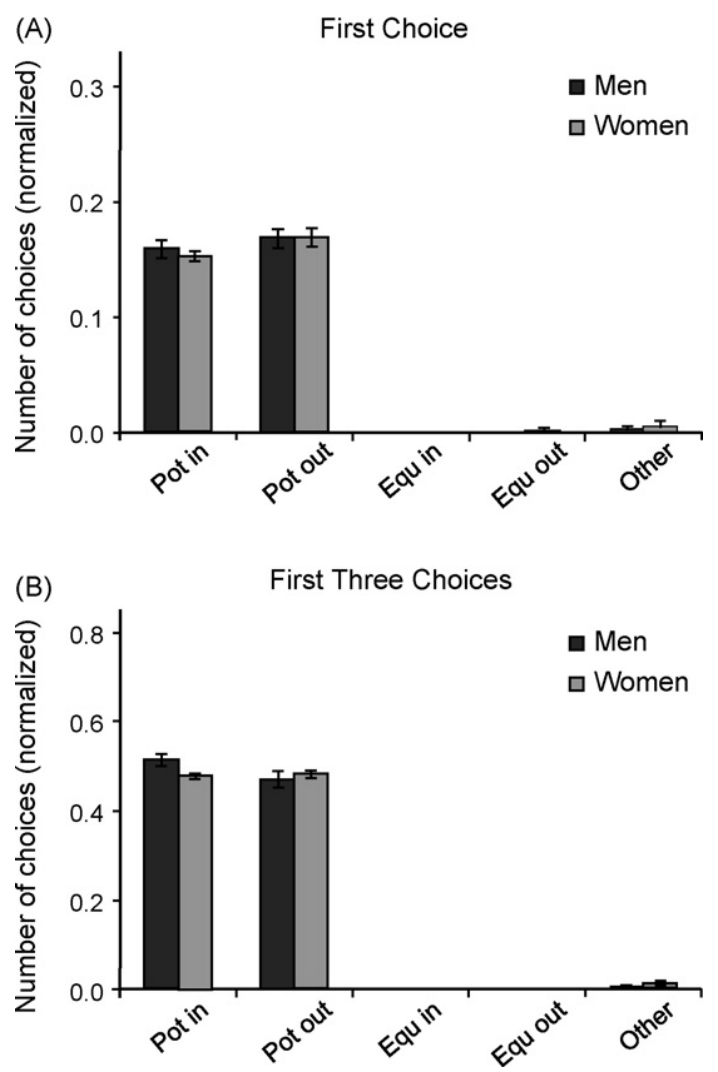

Fig. 4. Males' and females' choices in the local cue condition (Days 2 and 4 ) of Experiment 1. (A) First cup opened upon entering the arena. (B) First three cups opened upon entering the arena. The number of choices in each category $(n)$ is normalized according to the probability of making that choice ( $n / 3$ for Pot In, Pot Out, Equ In, Equ Out, and $n / 6$ for Other).
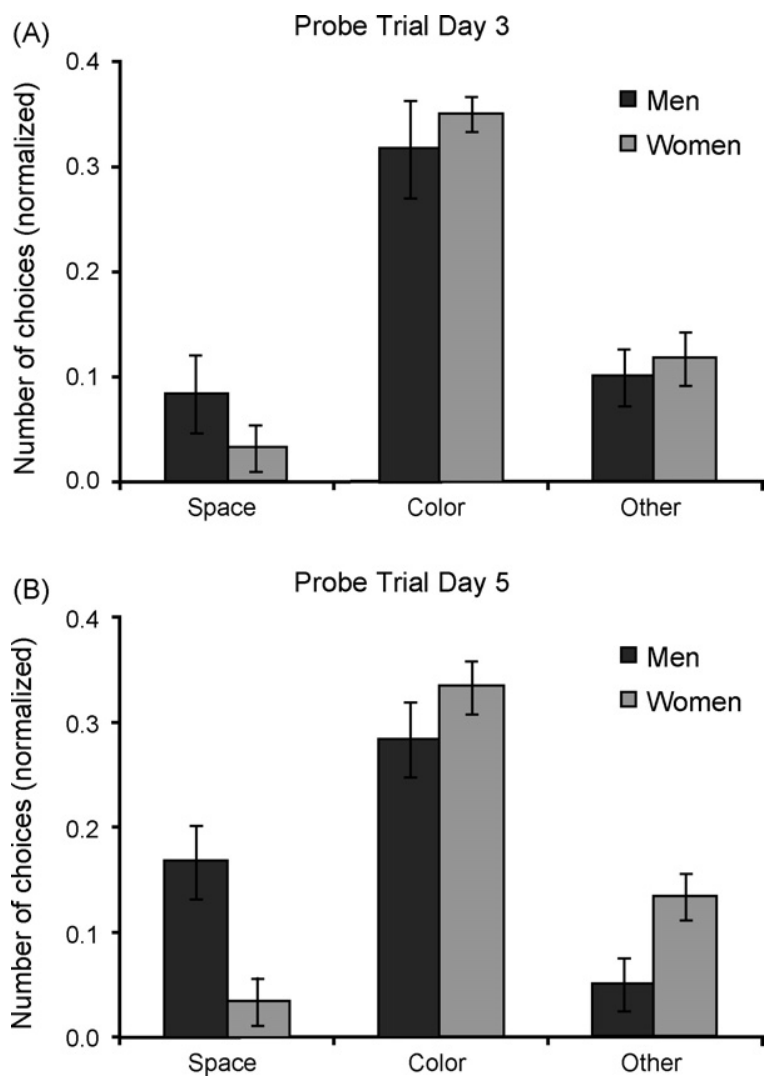

Fig. 5. Males' and females' choices during the dissociation probe trials on Day 3 (A) and Day 5 (B) of Experiment 1. First three cups opened upon entering the arena. Space: white cups at previously potentially-rewarded locations $(4,8,12,13,15$, 17). Color: colored cups at previously never-rewarded locations $(2,6,10,14,16$, 18). Other: white cups at never-rewarded locations $(1,3,5,7,9,11)$. There were no rewards present during the probe trials. The number of choices in each category $(n)$ is normalized according to the probability of making that choice ( $n / 6$ for Space, Color, Other).

rewarded correct spatial locations (Space; Fig. 5B; $F_{(2,18)}=29.077$, $P<0.001$, Color $>$ Other $>$ Space, all $P<0.024)$. Further analyses of the first six and all cups opened during the probe trial on Day 5 failed to reveal any female preference for correct spatial locations as compared to other never-rewarded locations (data not shown). The fact that a female preference for previously-rewarded spatial locations failed to develop can be explained by the fact that during the probe trials, after subjects had tested a few of the locations where they believed the rewards to be but were not rewarded, both males and females began to search in a systematic, but nonselective manner (e.g., in a circle around the arena) until they had opened most or all of the cups in the arena (statistical analyses showed that for the first six and all cups opened, males and females opened all types of cups with equal frequency; data not shown).

\subsubsection{First trial in the spatial relational condition}

The probe trial on Day 3 suggested that both males and females preferred to first search locations marked by a local cue (i.e., a colored cup). The probe trial on Day 5, however, suggested that female subjects did not remember the previously-rewarded spatial locations as well as the male subjects (i.e., females did not demonstrate a preference for correct spatial locations when local cues and spatial locations were incoherent). We therefore analyzed the first three choices that subjects made in the very first spatial relational trial on Day 3 (the subjects' first trial in the absence of local cues), as well as their first three choices in the first spatial relational trial on Day 5 (after subjects had experienced 1 day of testing in the absence of 

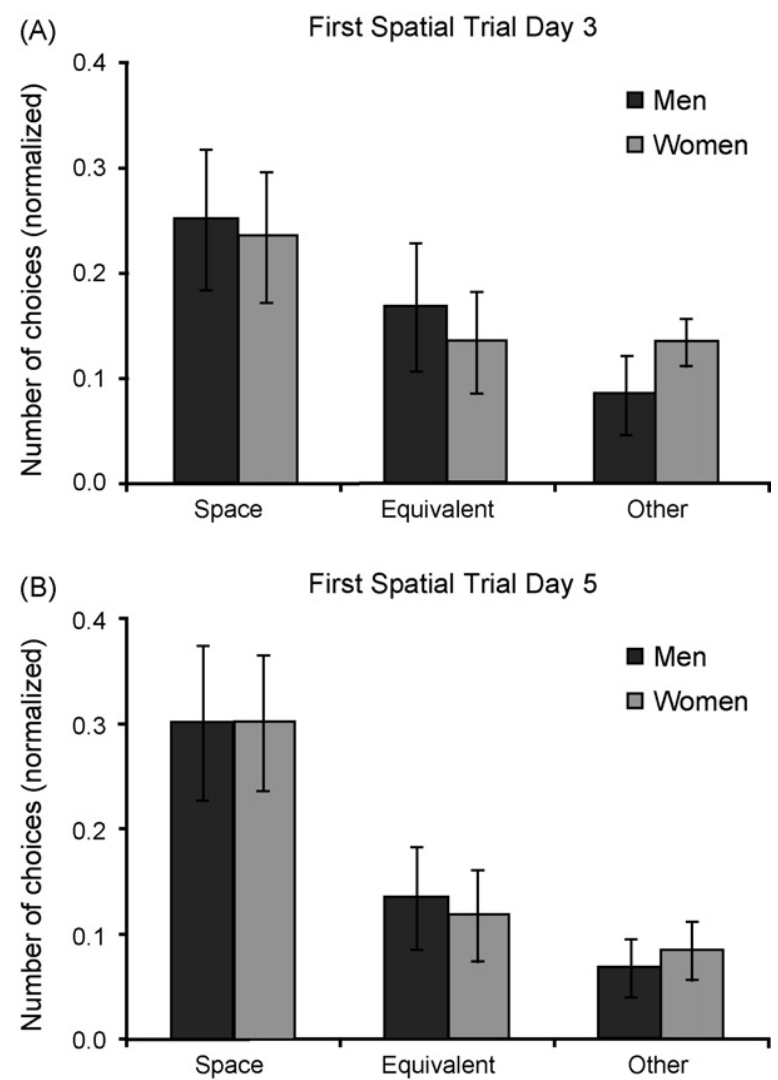

Fig. 6. Males' and females' choices on the first trial in the spatial relational condition on Day 3 (A), and Day 5 (B) of Experiment 1. First three cups opened upon entering the arena. Space: white cups at potentially-rewarded locations $(4,8,12,13,15,17)$. Equivalent: white cups at never-rewarded equivalent corner locations $(2,6,10,14$, $16,18)$. Other: white cups at never-rewarded locations $(1,3,5,7,9,11)$. Note that the categories 'Space' and 'Other' are the same as in Fig. 5. The category 'Equivalent' corresponds to the category 'Color' in Fig. 5, except for the fact that the cups were white and not colored for the trials in the spatial relational condition. The number of choices in each category $(n)$ is normalized according to the probability of making that choice ( $n / 6$ for Space, Equivalent, Other).

local cues, Day 3, followed by one additional day in the presence of local cues, Day 4). For this analysis, we designated three choice categories: (1) Space, the six potentially-rewarded locations; (2) Equivalent, the six never-rewarded cups at equivalent corner locations; and (3) Other, the six never-rewarded locations on the sides of the outer hexagon.

For the first three cups opened on the first spatial relational trial of Day 3, neither males nor females exhibited a preference for spatial locations as compared to equivalent locations (Fig. 6A; gender: $F_{(1,18)}=0.0, P=1.000$; cup choice: $F_{(2,36)}=2.293$, $P=0.116$; interaction: $F_{(2,36)}=0.240, P=0.788$; Space = Equivalent, $P=0.278$ ). Furthermore, although they chose spatial locations more often than Other locations (Space $>$ Other, $P=0.039$ ), they did not discriminate between equivalent locations and Other locations (Equivalent $=$ Other, $P=0.351$ ). In contrast, for the first three cups opened on the first spatial relational trial of Day 5, both males and females preferentially chose potentially-rewarded, correct spatial locations (Fig. 6B; gender: $F_{(1,18)}=0.000, P=1.000$; cup choice: $F_{(2,36)}=7.265, P=0.002$; interaction: $F_{(2,36)}=0.036$, $P=0.965$; Space $>$ Equivalent $>$ Other, all $P<0.044)$.

Thus, although females had failed to show a preference for the correct spatial locations during the probe trial on Day 5 (as described above), the immediately succeeding trial showed that they did indeed know, and could demonstrate a preference for, the correct spatial locations.

\subsubsection{Overall results in the spatial relational condition}

We further evaluated the ability of male and female subjects to discriminate the potentially-rewarded locations across all trials in the spatial relational condition. For these analyses, we combined the results for all of the trials that occurred in the absence of local cues on Days 3 and 5. For their first choice, both males and females chose potentially-rewarded locations more often than never-rewarded locations (Fig. 7A; gender: $F_{(1,18)}=0.634, P=0.436$; cup choice: $F_{(4,72)}=198.387, P<0.0001$; interaction: $F_{(4,72)}=0.546$, $P=0.703$; Pot IN $=$ Pot OUT $>$ Equ IN $=$ Equ OUT, all $P<0.0001$; Equ IN $>$ Other, $P=0.049$; Equ OUT = Other, $P=0.209$ ). Similarly, for their first three choices both males and females continued to choose potentially-rewarded locations more often than never-rewarded locations (Fig. 7B; gender: $F_{(1,18)}=0.014, P=0.908$; cup choice: $F_{(4,72)}=328.384, P<0.0001$; interaction: $F_{(4,72)}=0.004, P=1.000$; Pot IN $=$ Pot OUT $>$ Equ IN $=$ Equ OUT $=$ Other, all $P<0.0001$ ). Analyses of each individual subject's choices in the spatial relational condition confirmed that all 20 subjects ( 10 males, 10 females) were equally motivated and discriminated the potentially-rewarded from the never-rewarded locations equally well (data not shown). Thus, in the spatial relational condition where subjects must rely on an allocentric, spatial relational representation of the environment in order to identify potentially-rewarded locations, both men and women discriminated the potentially-rewarded locations from the never-rewarded locations equally well.

\subsubsection{Non-spatial relational learning}

The presence of a centrally located object which was associated with the presence of rewards in either the inner or outer array of potentially-rewarded locations (Fig. 2A and C) allowed us
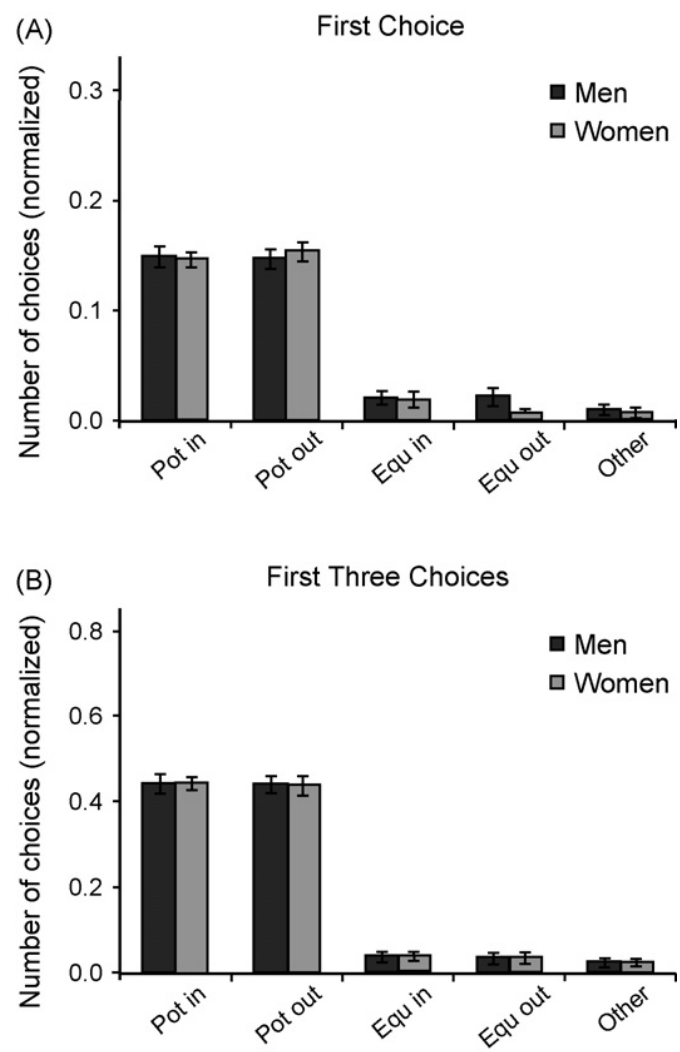

Fig. 7. Males' and females' choices in the spatial relational condition (Days 3 and 5) of Experiment 1. (A) First cup opened upon entering the arena. (B) First three cups opened upon entering the arena. The number of choices in each category $(n)$ is normalized according to the probability of making that choice $(n / 3$ for Pot In, Pot Out, Equ In, Equ Out, and $n / 6$ for Other). 
Table 1

Type of choices based on the predictor cues (central objects) in trials in which the rewarded locations were different from the preceding trial (Days 4 and 5).

\begin{tabular}{rlllll}
\hline Subject & Schedule & Gender & Choices & \\
& & & Chi & \\
& & & Correct & Incorrect & \\
\hline 1 & 1 & M & 1 & 3 & No \\
4 & 1 & M & 4 & 0 & Yes \\
6 & 1 & M & 4 & 0 & Yes \\
7 & 1 & M & 4 & 0 & Yes \\
17 & 1 & M & 4 & 0 & Yes \\
2 & 1 & F & 4 & 0 & Yes \\
3 & 1 & F & 4 & 0 & Yes \\
5 & 1 & F & 0 & 4 & No \\
16 & 1 & F & 4 & 0 & Yes \\
20 & 1 & F & 4 & 0 & Yes \\
11 & 2 & M & 7 & 1 & Yes \\
12 & 2 & M & 8 & 0 & Yes \\
13 & 2 & M & 5 & 2 & No \\
14 & 2 & M & 7 & 1 & Yes \\
19 & 2 & M & 8 & 0 & Yes \\
8 & 2 & F & 8 & 0 & Yes \\
9 & 2 & F & 8 & 0 & Yes \\
10 & 2 & F & 7 & 0 & Yes \\
15 & 2 & F & 7 & 0 & Yes \\
18 & 2 & F & 8 & 0 & Yes \\
\hline
\end{tabular}

to evaluate adult human subjects' ability to use non-spatial relational information to guide their choices. Specifically, for Days 1 , 2 and 3, the presence of a green rectangular bucket indicated that the rewarded locations could be found on the inner array (Fig. 2: locations $13,15,17)$, at unique spatially-defined locations. In contrast, the presence of a yellow cylindrical bin indicated that the rewarded locations could be found on the outer array, again in their unique, spatially-defined locations (Fig. 2: locations 4, 8, 12). The first evidence as to whether subjects were using the central object came from the first trial of Day 4 in which the relationship between the central object and the rewarded array was reversed as compared to the first three days (i.e., the green central object was subsequently associated with the presence of rewards on the outer array, and the yellow central object associated with rewards on the inner array). Out of 20 subjects, 15 (75\%) chose the unrewarded array on this trial, demonstrating at the population level $\left(\mathrm{Chi}^{2}=5.00, P<0.05\right)$ that the subjects were using non-spatial relational information (the central objects) to guide their choices in a real-world, open-field environment. Interestingly, when tested with this same experimental paradigm, neither squirrels [30] nor monkeys [24] demonstrated this ability.

In order to further evaluate whether individual subjects relied on the central object to predict rewarded locations, we analyzed their first choice upon entering the arena on the specific trials of Days 4 and 5 during which the central object and the rewarded array were different from those used in the immediately preceding trial (and thus excluding all trials in which the central object and rewarded array were the same as in the preceding trial). We performed Chi-square analyses on the number of correct versus incorrect choices (Table 1). Our analyses showed that 17 of the 20 subjects ( 9 females and 8 males) used the central object to guide their choices (all $\mathrm{Chi}^{2}$ for the 17 subjects $>3.841, P<0.05$ ). Of the three subjects that did not use the central object, two (female subject 5 , and male subject 1 ) consistently went back to the previously-rewarded array (Table 1 ). Thus, for example, if the inner array was rewarded on the first trial, these subjects would invariably choose a potentially-rewarded location on the inner array for their first choice of the second trial. If, upon checking the inner array, these subjects did not find a reward, they would then shift their search to the opposite array, i.e., the outer array in this example. In post-experiment questioning (conducted immediately after their last trial on their last testing day), these two subjects con- fessed that they had no idea that the central objects served any other purpose than to mark the center of the arena.

The second male subject (subject 13) who did not exhibit statistical evidence of using the central object to guide his choices told the experimenter (PBL) after his last trial on Day 4 that he had just recognized that there was a consistent relationship between the central object and the rewarded array (NB: this subject was not questioned in any manner, but rather felt compelled to relate his realization to the experimenter). Nevertheless, this subject made several mistakes on the final day of testing with respect to correlating the central object and the rewarded array, and thus the statistical analysis of his choices was unable to verify that he was indeed using the central object to guide his choices.

\subsection{Experiment 2}

\subsubsection{Number of locations searched}

For this experiment we were interested in testing male and female subjects with three pseudo-randomly arranged reward locations among the 18 possible locations. We first analyzed the total number of locations searched (i.e., foot pads stepped on) by male and female subjects in order to detect potential gender differences in overall task performance. In contrast to Experiment 1 where inverted cups hid the rewards, in Experiment 2 where three asymmetrically-located illuminating foot pads served as goal locations, we did not find any difference between the total number of locations searched by males and females (Fig. 8A) in either the 13 local cue trials (odd-numbered trials 1, 3, 5, ., 25; gender: $F_{(1,16)}=0.18, P=0.6797$; trials: $F_{(12,192)}=13.91, P<0.0001$; interaction: $F_{(12,192)}=0.46, P=0.9365$; males: $6.33 \pm 0.57$ locations; females: $7.42 \pm 0.65$ locations), nor in the 13 spatial relational trials (even-numbered trials $2,4,6, \ldots, 26$; gender: $F_{(1,16)}=0.39, P=0.5423$; trials: $F_{(12,192)}=11.87, P<0.0001$;
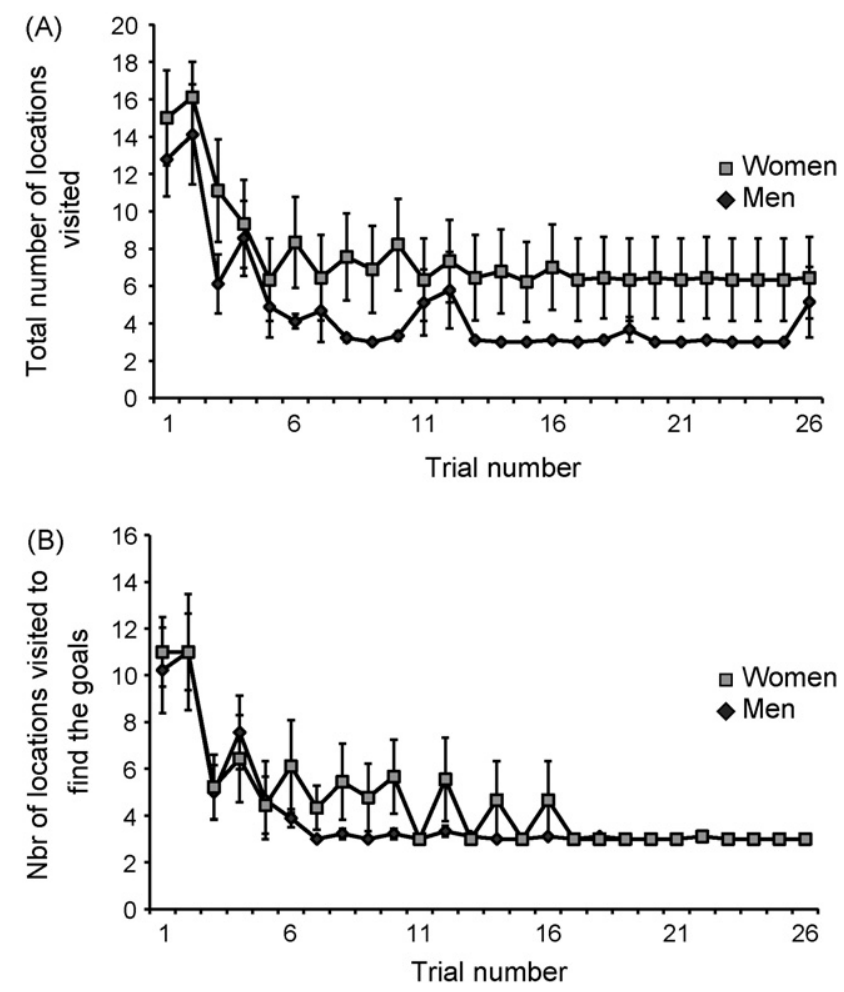

Fig. 8. Number of locations searched by males and females in the local cue (trials $1,3,5, \ldots$ ) and spatial relational (trials $2,4,6, \ldots$ ) conditions of Experiment 2 . (A) Total number of locations visited (i.e., foot pads touched) per trial. (B) Number of locations visited to find the three illuminating foot pads per trial. 
interaction: $F_{(12,192)}=0.70, P=0.7533$; males: $6.30 \pm 0.56$ locations; females: $7.91 \pm 0.64$ locations). Nevertheless, 2 (of 9) female subjects persisted in checking all 18 locations for all 26 trials, resulting in a larger mean and variation in the total number of locations searched by females. None of the nine male subjects ever persisted in checking every location on every trial.

Consistent with Experiment 1, males and females did not differ in the number of locations searched to find the three goal locations. In order to focus on the learning curve, we compared the number of locations searched to find the three goals by males and females for the first eight local cue and the first eight spatial relational trials (before an asymptotic level of performance was reached), but found no difference between genders (Fig. 8B, local cue condition, gender: $F_{(1,16)}=0.31, P=0.5843$; trials: $F_{(7,112)}=17.48$, $P<0.0001$; interaction: $F_{(7,112)}=0.37, P=0.9160$; males: $6.94 \pm 0.77$ cups; females: $8.83 \pm 0.84$ cups; spatial relational condition, gender: $F_{(1,16)}=0.87, P=0.3645$; trials: $F_{(7,112)}=11.21, P<0.0001$; interaction: $F_{(7,112)}=0.77, P=0.6127$; males: $4.79 \pm 0.48$ cups; females: $6.19 \pm 0.62$ cups). An expanded analysis of all trials failed to find any gender differences in the number of locations searched in order to find the three goal locations neither for the 13 local cue trials (Fig. 8B, gender: $F_{(1,16)}=0.31, P=0.5843$; trials: $F_{(12,192)}=16.96, P<0.0001$; interaction: $F_{(12,192)}=0.36, P=0.9747$; males: $3.85 \pm 0.27$ locations; females: $4.14 \pm 0.29$ locations), nor the 13 spatial relational trials (gender: $F_{(1,16)}=0.85, P=0.3690$; trials: $F_{(12,192)}=11.03, P<0.0001$; interaction: $F_{(12,192)}=0.81, P=0.6391$; males: $4.12 \pm 0.31$ locations; females: $4.97 \pm 0.41$ locations).

\subsubsection{Local cue condition}

We then evaluated the subjects' ability to discriminate the three asymmetrically-arranged goal locations in the presence of local cues marking these locations (i.e., colored rings that surrounded the target foot pads). We analyzed the first 3 locations subjects chose when entering the arena during the last 10 local cue trials (i.e., trials 7, 9, 11, ., 25). Both males and females choose rewarded locations more often than neverrewarded locations (Fig. 9A; gender: $F_{(1,16)}=0.05, P=0.8179$; location choice: $F_{(1,16)}=23297.06, P<0.0001$; Correct $>$ Incorrect; interaction: $\left.F_{(1,16)}=0.12, P=0.7361\right)$. Analyses of each individual subject's choices in the local cue condition confirmed that all 18 subjects were equally motivated and discriminated the potentiallyrewarded from the never-rewarded locations (data not shown). In sum, in the local cue condition, all subjects readily discriminated the three goal locations marked by local cues. Both male and female subjects were highly selective and chose almost exclusively locations that were marked with local cues.

\subsubsection{Spatial relational condition}

We evaluated the subjects' ability to discriminate the three asymmetrically-arranged goal locations in the absence of local cues marking these locations. We analyzed the first 3 locations subjects chose when entering the arena during the last 10 spatial relational trials (i.e., trials $8,10,12, \ldots, 26$ ). Again, both males and females choose rewarded locations more often than never-rewarded locations (Fig. 9B; gender: $F_{(1,16)}=0.14, P=0.7135$; location choice: $F_{(1,16)}=1496.05, P<0.0001$; Correct $>$ Incorrect; interaction: $\left.F_{(1,16)}=0.18, P=0.6740\right)$. Analyses of each individual subject's choices in the spatial relational condition confirmed that all 18 subjects were equally motivated and discriminated the potentially-rewarded from the never-rewarded locations (data not shown). In sum, in the spatial relational condition, all subjects readily discriminated the three goal locations in the absence of local cues. Both male and female subjects were highly selective and chose goal locations almost exclusively.
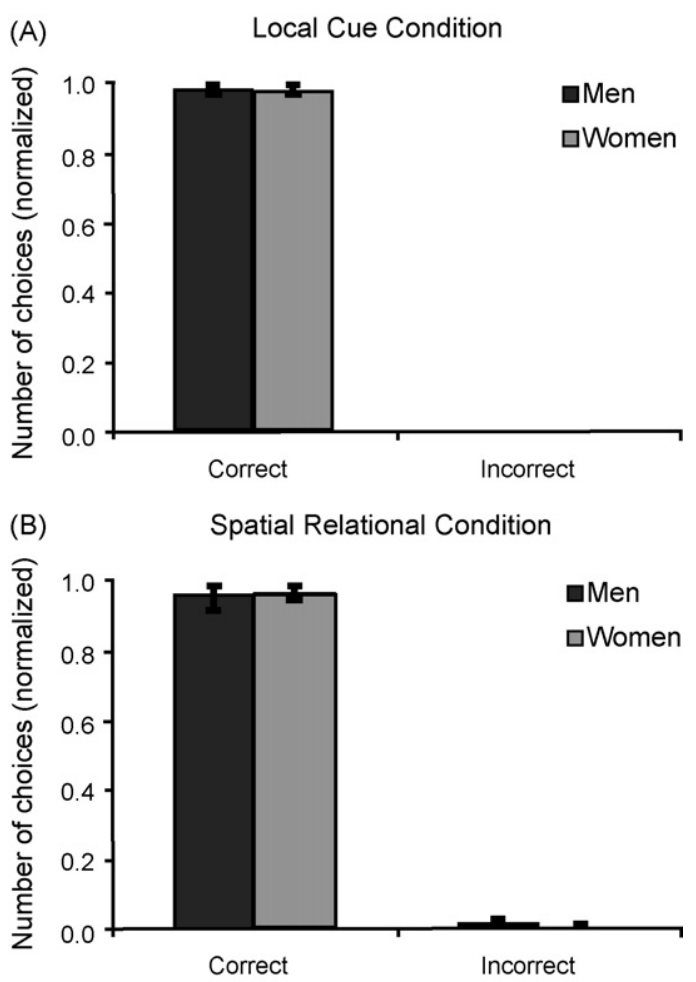

Fig. 9. Males' and females' choices in the local cue (A) and spatial relational (B) condition of Experiment 2. First three locations visited upon entering the arena.

\subsubsection{Probe trials}

The 27th and last trial of testing was a probe trial during which the colored rings were shifted from locations 7, 10 and 14 to locations 3, 12 and 17. As in Experiment 1, the aim of this probe trial was to assess subject reliance on local versus spatial relational information to discriminate rewarded locations from non-rewarded locations. As before, location choices were classified into one of three categories: "Space" designated the three previously-rewarded locations (although the foot pads would not light up during the probe trial), "Color" designated the three locations now encircled by colored rings, and "Other" designated the twelve locations that were never rewarded and not covered by colored rings.

Individual analyses of the first location searched during the probe trial (data not shown) revealed that five males chose neutral foot pads at previously-rewarded correct spatial locations (Space) and four males chose foot pads encircled by a colored ring at incorrect spatial locations (Color); three females chose Space locations, five females chose Color locations and one female chose an Other location. Therefore, at the group level, males and females did not differ in their preference for Space and Color locations, and chose both Space and Color locations significantly more than Other locations (gender: $F_{(1,16)}=1.0, P>0.3322$; locations: $F_{(2,32)}=4.78, P<0.0153$; Space $=$ Color $>$ Other, all $P<0.018$; interaction: $\left.F_{(2,32)}=0.51, P=0.6076\right)$.

For the first three locations searched during the probe trial, again males and females did not differ (Fig. 10; gender: $F_{(1,16)}=2.29$, $P>0.1578$ ), and both sexes exhibited equal preference for Space and Color locations, and chose both Space and Color locations significantly more than Other locations (Fig. 10; locations: $F_{(2,32)}=29.81, P<0.0001$; Space $=$ Color $>$ Other, all $P<0.0001$; inter action: $\left.F_{(2,32)}=0.50, P=0.6139\right)$. In sum, after being tested for 26 trials in alternating, local cue and spatial relational conditions, both men and women gave similar weight to local and spatial information, even though spatial relational information was the only consistently (i.e., at every trial) valid information to discriminate 


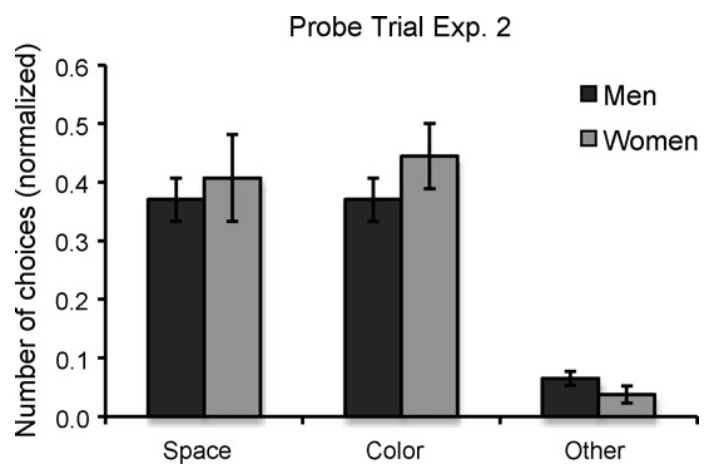

Fig. 10. Males' and females' choices in the dissociation probe trial (trial 27). Space: grey, non-illuminating foot pads at previously illuminating locations 7, 10 and 14, no local cues present. Color: grey foot pads marked with a colored plastic disk at locations 3,12 and 17. Other: grey unmarked foot pads at never-illuminating locations $(1,2,4,5,6,8,9,11,13,15,16,18)$. No foot pads illuminated during the probe trial. The number of choices in each category $(n)$ is normalized according to the probability of making that choice ( $n / 3$ for Space and Color, $n / 12$ for Other).

the goal locations. In Experiment 2, as in Experiment 1, men and women exhibited similar spatial learning and memory abilities in this real-world, open-field environment.

\section{Discussion}

The aim of this study was to assess possible gender differences in spatial and non-spatial relational learning and memory in normal adult human subjects behaving freely in a controlled, real-world, open-field environment. In Experiment 1, we found that: (1) females exhibited more exploratory behavior during the initial phase of testing; (2) both males and females discriminated the potentially-rewarded locations equally well in the presence of local visual cues; (3) when the local and spatial information conflicted in predicting the rewarded locations, males considered both local visual and spatial relational information, whereas females preferred local visual information and ignored the spatial relational information, and (4) in the absence of local visual cues, females discriminated the potentially-rewarded locations as well as males.

Experiment 2, employing a different array of rewarded locations, confirmed our primary finding from Experiment 1 that male and female subjects did not differ in their ability to identify the goal locations in the absence of local cues. Although, as a group, females in Experiment 2 did not demonstrate increased exploratory behavior, 2 of the 9 females nonetheless persisted in checking all 18 locations for all 26 trials. No male ever exhibited such behavior. Experiment 2 also failed to reveal a differential preference for local versus spatial information between males and females during the probe trial, which followed 26 trials in alternating conditions (local cue and spatial relational). We discuss in detail below how paradigm differences between Experiments 1 and 2 might have influenced individual subjects' behavior.

In sum, although particular aspects of task performance may have differed between genders, our experiments provide no evidence that women have poorer spatial relational learning and memory abilities than men in a controlled, real-world open-field environment. We discuss our findings with respect to previous virtual reality experiments which reported superior spatial abilities in men as compared to women.

\subsection{Gender-specific strategies in our real-world, open-field environment}

\subsubsection{Exploratory behavior}

The first indication that females approached the task differently than males came from the total number of cups opened in
Experiment 1 (Fig. 3A). On Day 1 (but not on Days 2-5), females opened overall more cups than males, and simply constraining our analysis to this variable would lead to the conclusion that females were slower than males to learn about the task, or that they discriminated the rewarded locations less well (even in presence of local cues). However, there was no difference between males and females in the number of cups opened in order to find all three rewards on the first day (Fig. 3B). Rather, the difference between males and females in the number of cups opened was evident only after the third reward was found, with females opening more cups. Other studies have shown increased exploratory behavior by females, as compared to males, in different species and with different paradigms. For example, when placed in openfield environments female rats exhibit increased locomotor and exploratory behavior as compared to male rats [17,22]. More relevant is an investigation of wayfinding abilities in men and women in an indoor environment that noted significantly more "exploratory action" on the part of female subjects during reverse wayfinding [31].

The underlying reasons for the observed increased exploratory behavior are unclear. It may result, for example, from a lack of trust in the experimenter, or lower self-confidence or confidence in their knowledge. In the first case, females might be less trusting, or more skeptical, with respect to the intentions of the experimenter or the goal of the experiment, and thus less-inclined to believe that the rules of the task would not change from one trial to the next. Males possibly believed that they had ascertained the goal of the experiment, trusted that the succeeding trials would be similar to the preceding trials, and thus felt there was no need to continue exploring. Females, on the other hand, might have felt compelled to continue verifying the rules of the task, or to verify that the experimenter was not trying to trick them.

In the second case, numerous sociobiological studies have identified female confidence issues even in university and postgraduate (e.g., medical) students [8-11]. Lower self-confidence is particularly evident when females are tested on subject matter that is judged a priori to be male-typed (i.e., tasks, like spatial competence, where males are typically expected to outperform females [8-10]). Thus, even if females had ascertained the goal of the experiment, they might not have trusted their own assessment, and therefore perseverated in searching until they had confirmed that they had identified all of the goal locations.

In Experiment 2, local cues provided confirmation of the spatial location of the goals on every other trial. As has been previously shown in rats in a similar open-field environment, the presence of local cues can reduce the number of non-rewarded locations searched without necessarily impacting the spatial relational memory of the goal locations [26]. Although females' tendency to explore might have been reduced by the specific (alternating) training conditions in Experiment 2, 2 of the 9 females nonetheless persisted in searching all 18 locations for all 26 trials. Similarly, in Experiment 1, 1 female persisted in opening all 18 cups for all 50 trials. In contrast, no males ever exhibited this behavior. When these female subjects were questioned after the experiment as to why they persisted in checking all of the locations, all three responded that they could not be sure that the rules of the game would not change from trial-to-trial, and that the only way to be certain was to check every location.

\subsubsection{Preference for local cues}

Female subjects also behaved differently from males on the second probe trial (Day 5) of Experiment 1 where local cue information conflicted with the spatial information. Whereas men considered both local and spatial relational information, women 
demonstrated a preference only for the local cue information and failed to discriminate the previously-rewarded spatial locations from never-rewarded ("Other") locations. If considered alone, this finding would suggest that females did not know the correct spatial locations. Results of the immediately following trial in the spatial relational condition, however, revealed that, in the absence of local cues, women discriminated the goal locations as well as men. Possibly, women considered that when local cues were present, they were a necessary confirmation of the presence of a reward. Thus, even though they knew the position of the rewarded locations as well as men, they refrained from opening cups in the absence of local confirmation. Indeed, the presence of local olfactory or visual cues can influence the choices of rats when tested in a similar open-field arena, causing them to become much more selective and reducing their search among non-marked locations $[26,28]$.

In Experiment 2, subjects were exposed to an alternating testing schedule (i.e., one local cue trial, one spatial relational trial, etc.) and on the probe trial performed at the end of testing, men and women did not differ in their preference for local or spatial relational information. The alternating trials of Experiment 2 may have either reduced the confirmational weight of the local cues, or emphasized the fact that spatial relational information was the only consistently relevant information that could be used to discriminate the goal locations on every single trial.

In sum, the results of probe trials must always be interpreted with caution as they provide only a partial indication of the subjects' knowledge (e.g., the relative weight given to different sources of information during decision-making). As such, their results do not necessarily reflect the strength of the memory for the particular class of stimuli under investigation.

\subsection{Are there gender differences in spatial relational learning and memory?}

There is a large literature describing male superiority in spatial abilities, as assessed by mental rotation tasks $[1,7,15,40,42,50,53]$ (see [41] for a review), wayfinding tasks [16,31,43,44,47], and environmental pointing tasks $[7,23]$. Interestingly, the sole commonly used spatial task where women outperform, or at least do not always underperform as compared to males, are some versions of the object-in-location task $[43,50]$, but see $[19,43]$. These tasks, however, differ fundamentally from our test of allocentric, spatial relational learning and memory. Wayfinding and environmental pointing tasks, for example, are complex tests of spatial abilities, in which subject performance may be influenced by other psychological processes that are themselves sexually dimorphic [8-10,32]. Mental rotation tasks do not test allocentric spatial memory and accordingly do not activate medial temporal lobe structures in order to solve them [21]. Similarly, the object-in-location task is typically administered on a piece of paper or a computer screen and has, therefore, a very strong egocentric component making a direct comparison with our task inappropriate [5,43]. Consequently, we restrict our discussion to studies employing tasks that were designed to test allocentric spatial memory in adult humans.

Our results indicate that even though men and women may exhibit different strategies when solving a real-world spatial relational memory task, and may even give different weights to various environmental cues, they did not differ in their overall ability or accuracy to solve the task. Interestingly, a detailed review of previous studies suggesting gender differences in allocentric spatial relational memory, especially in light of the current findings, can lead to the same conclusion. Although many studies have documented differences in how men and women perform these spatial tasks $[2,3,7,12,33,36,46,47]$, they have not necessarily demonstrated gender differences in spatial memory specifically.

\subsection{Evaluation of experimental indices of memory}

\subsubsection{Latency and path distance to find the target location}

Current paradigms for studying gender differences in spatial relational memory abilities include the Morris search task (a.k.a., Morris water maze) in a virtual reality environment $[2,3,36,46]$ as well as the traditional labyrinth-style maze also presented in a virtual environment [33]. Subjects' performance in the Morris search task is typically assessed by measuring the latency and path distance to find the target location during training trials, as well as, during probe trials (in which the hidden platform is not revealed when traversed), the heading error towards the platform location and the percentage of time spent in the quadrant of the pool in which the platform was previously located. In the labyrinth-style maze, subjects' performance is evaluated by the latency to finish the maze, and the number of errors (i.e., turns into dead-end alleys, or doubling back in correct alleys) committed by subjects. These studies have found that during individual trials women consistently take longer to find the hidden platform or to solve the maze than men, and that in the Morris search task their paths to find the platform are typically longer than those of men. These results have lead to the conclusion that females must not know the layout of the virtual arena as well as males, or that their spatial memory is less proficient. In our study, we found that on the first day (i.e., the first 10 trials), women explored the arena more than men. Nevertheless, despite their increased exploratory behavior, women did not prove to be any less accurate in our spatial task: females first identified the rewarded locations, and then continued exploring the arena. If subjects had not been allowed to explore the environment after finding the rewards, some might have done so before focusing on the experimenter-defined 'goal' of the task [27,29]. Under such conditions, females' increased exploratory behavior might have thus led to an increase in the number of "errors" prior to finding all the rewards, resulting in poorer performance on the task as compared to males. In the virtual Morris search task, increased exploratory behavior could directly translate into increased latencies and swim paths. In virtual mazes, increased exploratory behavior could lead to increases in latency and number of errors. These differences in task performance, however, especially in the absence of further corroborating evidence, cannot necessarily be interpreted as evidence of gender differences in spatial memory per se [13,45,52].

Females are also generally slower than males when navigating, whether in virtual reality or in the real world. In virtual environments, studies employing the Morris search task have shown that women are often slower than men even when the platform is visible ([2], $P=0.096$ in a two-tailed $t$-test; [36], $P<0.05$ ). Moreover, Sandstrom et al. [46], showed that females were significantly slower than males at completing a pre-testing obstacle course that was intended to familiarize subjects with the virtual environment and test for any pre-existing differences in joystick ability or perception of the environment $(P<0.01)$. Interestingly, in studies using the virtual Morris search task paradigm, latency and swim path length decrease for both males and females as training proceeds $[2,36]$. Similarly, in virtual mazes, latency and the number of errors committed decrease for both males and females as training continues [33]. These decreases in latency might be the result of decreased exploratory behavior with increased experience in the environment. In our own study, subjects performed either 10 or 15 trials per day and a gender difference in exploratory behavior was evident only on Day 1. Thus, it is possible that if the aforementioned virtual reality studies had included longer periods of training (i.e., more than the 6-20 trials typically used), females' latency, path lengths or errors might decrease concomitantly with a decrease in exploratory behavior to equate to those of males at the end of training. One could argue, then, that the absence of gender differences would be due to a ceiling effect. Nevertheless, we contend that 
any measures that might be impacted by differences in exploratory behavior, as was shown for the studies described above, are necessarily inconclusive and insufficient by themselves to demonstrate gender differences in spatial memory, rather than simply differences in task performance $[13,45,52]$.

\subsubsection{Probe trials}

Given the possible limitations of the evidence obtained during regular trials, researchers employing the Morris search task generally also perform probe trials during which the reward or platform normally present at the goal location is absent. Although this trial is fundamentally different from standard trials, performance on probe trials might also be impacted by factors other than the memory for the spatial location of the goal per se.

The initial heading at which a subject departs when navigating to a hidden goal location seems to be both an intuitive and informative measure of spatial competence. However, this measure can also be biased by the strategy that the subject uses to navigate. For example, if a subject relies preferentially on geometric information to determine the general direction to the goal (e.g., as has been suggested for men; see [50] for a review) and thus heads directly toward the goal, then the initial heading is an informative measure with respect to spatial memory. However, if the subject needs to determine or confirm the direction to the goal in relation to specific landmarks in the environment (e.g., as has been suggested for women), then initial heading is not necessarily informative. Given the above-mentioned differences in exploratory strategies exhibited by men and women, the direction in which subjects head at the beginning of a trial might therefore be much less associated with their knowledge of a given spatial goal location than with the strategy they use to navigate to that location.

During the probe trial of the Morris search task, positive evidence that the subject knows and remembers the goal location is obtained when a subject continues to look for the platform in a location where he/she believes it to be, even in the absence of that goal (i.e., in the probe trial, the hidden platform is not revealed when the subject traverses its previous spatial location). A simple real world example of the Morris search task probe trial would be if one were to search for one's keys in the drawer where they are always kept, but not find them. The Morris search task would consider the subject that perseverates in looking in the drawer even when the keys are clearly not there as being more knowledgeable than the subject who, after not finding the keys there, searches elsewhere. In sum, whereas the amount of time spent in the target quadrant or the number of times the goal location is crossed during probe trials can be considered a valid estimation of perseverative behavior, it is not necessarily indicative of the strength or accuracy of a subject's spatial memory. In contrast, a simple modification to this probe trial, or the addition of a supplementary probe trial, where subjects are informed that in the subsequent trial the platform would not be present, but that they are to go to the location where the platform was habitually located, stop there, and remove their hand from the joystick, would be a valuable addition to this paradigm. The distance between the usual platform location and each individual's stopping point could then be calculated to assess potential gender differences in spatial memory accuracy under these conditions.

\subsection{Virtual versus real-world environments}

It is important to consider the possibility that males and females integrate information from the various sensory modalities differentially when constructing an allocentric, spatial relational representation of an environment. As discussed previously, realworld environments allow subjects access to nearly $180^{\circ}$ field of view without even turning their heads, as well as visual flow, vestibular, proprioceptive, somesthetic, and auditory information with which to elaborate their representation of the environment. It is possible that either the same or different brain areas in men and women utilize these various sensory inputs differentially $[6,18]$, for example by giving different weights to different sensory modalities. Interestingly, a series of studies by Tan et al. [51] showed that the use of a large, curved computer screen that offers $\sim 74^{\circ}$ display field of view can improve the performance of both men and women (as measured by latency and number of errors) in a virtual reality maze. Importantly, however, women's performance improvement was greater than that of men, and was, in fact, so great that any difference between men and women was eliminated when using this large screen. Their experiments showed that this improved female performance was specifically due to the increased optical flow information offered by the larger screen [51]. Thus, simply increasing the amount of visual flow available to female subjects can render female performance equivalent to that of males in this virtual task. What, then, are the consequences of depriving male and female subjects of information from the other sensory modalities that are normally present in real-world environments, and might this deprivation affect males and females differentially? Given the results of Tan et al. [51] it is possible that the asserted differences in the spatial memory abilities of male and female subjects are, rather, differences in visual information processing that are revealed under highly controlled conditions such as virtual reality experiments. Such differences were not found in the current experiment performed in a real-world, open-field environment in which information from all sensory modalities were available to contribute to the building of a spatial representation of the world.

\section{Conclusion}

A variety of evidence from a number of research avenues suggests that female performance in spatial tasks may be influenced by gender-specific differences in visual processing [51], strategy [12], and self-confidence [8-10]. A critical evaluation of earlier studies indicates that previously-reported gender differences in spatial task performance might be influenced by such factors, and therefore do not necessarily reflect gender differences in spatial memory per se. In our study performed in a controlled, real-world, openfield environment, men and women with full access to information from all sensory modalities did not differ in their allocentric, spatial relational learning and memory abilities.

\section{Acknowledgements}

This research was supported by the Swiss National Science Foundation: grant PMPDP3-122844 to PBL and grant PP00A106701 to PL.

\section{References}

[1] Albaret JM, Aubert E. Etalonnage 15-19 ans du test de rotation mentale de Vandenberg. Evolutions Psychomotrices 1996;8(34):206-15.

[2] Astur RS, Ortiz ML, Sutherland RJ. A characterization of performance by men and women in a virtual Morris water task: a large and reliable sex difference. Behavioural Brain Research 1998;93(1-2):185-90.

[3] Astur RS, Tropp J, Sava S, Constable RT, Markus EJ. Sex differences and correlations in a virtual Morris water task, a virtual radial arm maze, and mental rotation. Behavioural Brain Research 2004;151(1-2):103-15.

[4] Banta Lavenex P, Amaral DG, Lavenex P. Hippocampal lesion prevents spatial relational learning in adult macaque monkeys. Journal of Neuroscience 2006;26(17):4546-58.

[5] Banta Lavenex P, Lavenex P. Spatial memory and the monkey hippocampus: not all space is created equal. Hippocampus 2008;19(1):8-19.

[6] Bell EC, Willson MC, Wilman AH, Dave S, Silverstone PH. Males and females differ in brain activation during cognitive tasks. Neuroimage 2006;30(2):529-38.

[7] Bell S, Saucier D. Relationship among environmental pointing accuracy, mental rotation, sex, and hormones. Environment and Behavior 2004;36(2):251-65.

[8] Beyer S. Gender differences in self-perception and negative recall biases. Sex Roles 1998;38:103-33. 
[9] Beyer S. Gender differences in the accuracy of self-evaluations of performance. Journal of Personality and Social Psychology 1990;59(5):960-70.

[10] Beyer S, Bowden EM. Gender differences in self-perceptions: convergent evidence from three measures of accuracy and bias. Personality and Social Psychology Bulletin 1997;23(2):157-72.

[11] Blanch DC, Hall JA, Roter DL, Frankel RM. Medical student gender and issues of confidence. Patient Education and Counseling 2008;72(3):374-81.

[12] Brandner C. Strategy selection during exploratory behavior: sex differences. Judgement and Decision Making 2007;2(5):326-32.

[13] Cahill L, McGaugh JL, Weinberger NM. The neurobiology of learning and memory: some reminders to remember. Trends in Neurosciences 2001;24(10):578-81.

[14] Cattaneo Z, Postma A, Vecchi T. Gender differences in memory for object and word locations. Quarterly Journal of Experimental Psychology (Colchester) 2006;59(5):904-19.

[15] Cherney ID, Jagarlamudi K, Lawrence E, Shimabuku N. Experiential factors in sex differences on mental rotation. Perceptual and Motor Skills 2003;96(3 Pt 2):1062-70.

[16] Devlin AS, Bernstein J. Interactive wayfinding: use of cues by men and women. Journal of Environmental Psychology 1995;15:23-38.

[17] Goodrick CL. Free exploration and adaptation within an open field as a function of trials and between-trial-interval for mature-young, mature-old, and senescent Wistar rats. Journal of Gerontology 1971;26(1):58-62.

[18] Gron G, Wunderlich AP, Spitzer M, Tomczak R, Riepe MW. Brain activation during human navigation: gender-different neural networks as substrate of performance. Nature Neuroscience 2000;3(4):404-8.

[19] Iachini T, Sergi I, Ruggiero G, Gnisci A. Gender differences in object location memory in a real three-dimensional environment. Brain and Cognition 2005;59(1):52-9.

[20] Jarrard LE, Lewis TC. Effects of hippocampal ablation and intertrial interval on acquisition and extinction in a complex maze. American Journal of Psychology 1967;80(1):66-72.

[21] Jordan K, Wustenberg T, Heinze HJ, Peters M, Jancke L. Women and men exhibit different cortical activation patterns during mental rotation tasks. Neuropsychologia 2002;40(13):2397-408.

[22] Joseph R, Gallagher RE. Gender and early environmental influences on activity, overresponsiveness, and exploration. Developmental Psychobiology 1980;13(5):527-44

[23] Lambrey S, Berthoz A. Gender differences in the use of external landmarks versus spatial representations updated by self-motion. Journal of Integrative Neuroscience 2007;6(3):379-401.

[24] Lavenex P, Banta Lavenex P. Spatial relational memory in 9-month-old macaque monkeys. Learning and Memory 2006;13(1):84-96.

[25] Lavenex P, Banta Lavenex P. Amaral DG. Spatial relational learning persists following neonatal hippocampal lesions in macaque monkeys. Nature Neuroscience 2007;10(2):234-9.

[26] Lavenex P, Schenk F. Influence of local environmental olfactory cues on place learning in rats. Physiology and Behavior 1995;58(6):1059-66.

[27] Lavenex P, Schenk F. Integration of olfactory information in a spatial representation enabling accurate arm choice in the radial arm maze. Learning and Memory 1996;2(6):299-319.

[28] Lavenex P, Schenk F. Olfactory cues potentiate learning of distant visuospatial information. Neurobiology of Learning and Memory 1997;68(2): 140-53.

[29] Lavenex P, Schenk F. Olfactory traces and spatial learning in rats. Animal Behaviour 1998;56:1129-36.

[30] Lavenex P, Shiflett MW, Lee RK, Jacobs LF. Spatial versus nonspatial relational learning in free-ranging fox squirrels (Sciurus niger). Journal of Comparative Psychology 1998;112(2):127-36.

[31] Lawton CA, Charleston SI, Zieles AS. Individual and gender-related differences in indoor wayfinding. Environment and Behavior 1996;28(2):204-19.
[32] Malinowski JC, Gillespie WT. Individual differences in performance on a large-scale, real-world wayfinding task. Journal of Environmental Psychology 2001;21:73-82.

[33] Moffat SD, Hampson E, Hatzinpantelis M. Navigation in a "virtual" maze: sex differences and correlation with psychometric measures of spatial ability in humans. Evolution and Human Behavior 1998;19:73-87.

[34] Morris RG, Garrud P, Rawlins JN, O'Keefe J. Place navigation impaired in rats with hippocampal lesions. Nature 1982;297(5868):681-3.

[35] Morris RGM. Spatial localization does not require the presence of local cues. Learning and Motivation 1981;12(2):239-60.

[36] Mueller SC, Jackson CP, Skelton RW. Sex differences in a virtual water maze: an eye tracking and pupillometry study. Behavioural Brain Research 2008;193(2):209-15.

[37] O'Keefe J, Nadel L. In: Press C, editor. The hippocampus as a cognitive map. Oxford: Clarendon Press; 1978.

[38] Olton DS, Samuelson RJ. Remembrance of places passed: spatial memory in rats. Journal of Experimental Psychology: Animal Behavior Processes 1976;2:97-116.

[39] Olton DS, Walker JA, Wolf WA. A disconnection analysis of hippocampal function. Brain Research 1982;233(2):241-53.

[40] Peters M. Sex differences and the factor of time in solving Vandenberg and Kuse mental rotation problems. Brain and Cognition 2005;57(2):176-84.

[41] Peters M, Battista C. Applications of mental rotation figures of the Shepard and Metzler type and description of a mental rotation stimulus library. Brain and Cognition 2008;66(3):260-4.

[42] Peters M, Lehmann W, Takahira S, Takeuchi Y, Jordan K. Mental rotation test performance in four cross-cultural samples $(n=3367)$ : overall sex differences and the role of academic program in performance. Cortex 2006;42(7):1005-14.

[43] Postma A, Jager G, Kessels RP, Koppeschaar HP, van Honk J. Sex differences for selective forms of spatial memory. Brain and Cognition 2004;54(1):24-34.

[44] Prestopnik JL, Roskos-Ewoldsen B. The relations among wayfinding strategy use, sense of direction, sex, familiarity, and wayfinding ability. Journal of Environmental Psychology 2000;20:177-91.

[45] Rudy JW. The neurobiology of learning and memory. Sunderland, MA: Sinauer Associates, Inc.; 2008, 380 p.

[46] Sandstrom NJ, Kaufman J, Huettel SA. Males and females use different distal cues in a virtual environment navigation task. Brain Research Cognitive Brain Research 1998;6(4):351-60.

[47] Saucier DM, Green SM, Leason J, MacFadden A, Bell S, Elias LJ. Are sex differences in navigation caused by sexually dimorphic strategies or by differences in the ability to use the strategies? Behavioral Neuroscience 2002;116(3):403-10.

[48] Sautter CS, Cocchi L, Schenk F. Dynamic visual information plays a critical role for spatial navigation in water but not on solid ground. Behavioural Brain Research 2008;194(2):242-5.

[49] Schenk F, Grobéty M-C, Lavenex P, Lipp H-P. Dissociation between basic components of spatial memory in rats. In: Alleva E, Fasolo A, Lipp H-P, Nadel L, Ricceri $\mathrm{L}$, editors. Behavioural brain research in naturalistic and semi-naturalistic settings NATO ASI series, Series D, Behavioural and social sciences, vol. 82. The Netherlands: Kluwer; 1995. p. 277-300.

[50] Silverman I, Choi J, Peters M. The hunter-gatherer theory of sex differences in spatial abilities: data from 40 countries. Archives of Sexual Behavior 2007;36(2):261-8.

[51] Tan DS, Czerwinski MP, Robertson GG. Large displays enhance optical flow cues and narrow the gender gap in 3-D virtual navigation. Human Factors 2006;48(2):318-33.

[52] Tolman EC, Honzik CH. Introduction and removal of reward, and maze performance in rats. University of California Publications in Psychology 1930;4:257-75.

[53] Voyer D, Rodgers MA, McCormick PA. Timing conditions and the magnitude of gender differences on the Mental Rotations Test. Memory and Cognition 2004;32(1):72-82. 